\title{
Quantum magnetotransport properties of topological insulators under strain
}

\author{
M. Tahir and U. Schwingenschlögl* \\ PSE Division, KAUST, Thuwal 23955-6900, Kingdom of Saudi Arabia
}

(Received 26 June 2012; revised manuscript received 4 August 2012; published 15 August 2012)

\begin{abstract}
We present a detailed theoretical investigation of the quantum magnetotransport properties of topological insulators under strain. We consider an external magnetic field perpendicular to the surface of the topological insulator in the presence of strain induced by the substrate. The strain effects mix the lower and upper surface states of neighboring Landau levels into two unequally spaced energy branches. Analytical expressions are derived for the collisional conductivity for elastic impurity scattering in the first Born approximation. We also calculate the Hall conductivity using the Kubo formalism. Evidence for the beating of Shubnikov-de Haas oscillations is found from the temperature and magnetic field dependence of the collisional and Hall conductivities. In the regime of a strong magnetic field, the beating pattern is replaced by a splitting of the magnetoresistance peaks due to finite strain energy. These results are in excellent agreement with recent $\mathrm{HgTe}$ transport experiments.
\end{abstract}

PACS number(s): 73.20.At, 73.25.+i, 73.43.-f

\section{INTRODUCTION}

Recent progress in the realization of compounds with very strong spin-orbit interactions has attracted much interest in condensed matter physics. ${ }^{1-12}$ These compounds possess an energy gap in the bulk and gapless conducting states at the surface. They are known as topological insulators (TIs), exhibiting time-reversal symmetry. The surface states of $\mathrm{Bi}_{2} \mathrm{Se}_{3}, \mathrm{Sb}_{2} \mathrm{Te}_{3}$, and $\mathrm{Bi}_{2} \mathrm{Te}_{3}$ are two-dimensional nonideal Dirac fermions with a single Dirac cone. ${ }^{8}$ Dirac fermions have been observed in angle resolved photoemission experiments, and the quantization of the energy for the surface of TIs has been confirmed by scanning tunneling spectroscopy. ${ }^{11}$ The robust two-dimensional metal on the TI surface has conic branches touching at high symmetry points in the first Brillouin zone. ${ }^{8}$ The linear energy dispersion is responsible for common properties with graphene. ${ }^{2,4}$ However, there are also important differences. Nonideal Dirac-like surface bands in TIs arise from the mismatch of the bulk topological invariants on the two sides of the surface. The bulk remains in the topological phase. There is an odd number of not spin degenerate Dirac points at each surface. In addition, TIs exhibit a unique spin-momentum locking as a result of strong spin orbit interaction, which is essential for modeling topologically nontrivial insulators.

While so far the focus has been on the above mentioned compounds, recently growing attention is being paid to $\mathrm{HgTe}$ quantum wells, ${ }^{2,3}$ and transport measurements on two-dimensional HgTe have been reported. ${ }^{13}$ Due to the breaking of the inversion symmetry, three-dimensional $\mathrm{HgTe}$ is expected to have Dirac-like surface states. ${ }^{14,15}$ Different environments of the surface (upper surface) and the interface to the substrate (lower surface) break the inversion symmetry. The substrate makes it compulsory to include strain effects. In the presence of strain, a gap opens up and HgTe is expected to be a three-dimensional TI (Refs. 4 and 16). Recently, transport measurements of a thick $(70 \mathrm{~nm}) \mathrm{HgTe}$ sample in a perpendicular magnetic field (subject to strain effects) have been performed. ${ }^{17,18}$ The observed Rashba-like splitting has been explained by the breaking of the inversion symmetry in the external perpendicular magnetic field. Also, the Landau levels from the upper and lower surface states are found to remain degenerate as long as the hybridization between the two surface states is negligible. For a thickness of $70 \mathrm{~nm}$ this is the case since the width of the surface state is around 2 to $3 \mathrm{~nm}$. A complete quantum mechanical study of the transport properties of the surface states of TIs is needed to improve experiments and spintronic devices.

In this paper, we perform this study for an external perpendicular magnetic field including strain effects. The TI is considered to be thick enough that there is no substantial overlap of the wave functions of the upper and lower surfaces. Our focus is on the collisional magnetoconductivity in the presence of charged impurity scattering as well as the Hall conductivity in the standard Kubo formalism. Our results explain recent experiments on $\mathrm{HgTe}$, though they are general and can also be applied to $\mathrm{Bi}_{2} \mathrm{Se}_{3}, \mathrm{Sb}_{2} \mathrm{Te}_{3}$, and $\mathrm{Bi}_{2} \mathrm{Te}_{3}$, for example.

\section{RESULTS AND DISCUSSION}

We describe the surface states of HgTe by an effective Hamiltonian in the $x y$ plane. An external magnetic field $(0,0, B)$ is applied perpendicular to the TI surface under strain. The two-dimensional nonideal Dirac quasiparticle Hamiltonian in a magnetic field is ${ }^{17-19}$

$$
H=\tau_{z} v\left(\sigma_{x} \boldsymbol{\pi}_{y}-\sigma_{y} \boldsymbol{\pi}_{x}\right)+\tau_{z} \mathbf{I} \Delta
$$

Here $\mathbf{I}$ is the identity matrix, $\left(\sigma_{x}, \sigma_{y}, \sigma_{z}\right)$ is the vector of Pauli matrices in real spin space, and $\tau_{z}=+/-$ defines the upper and lower surfaces of the TI. Furthermore, $v$ denotes the Fermi velocity of the Dirac fermions, $\boldsymbol{\pi}=\mathbf{p}+e \mathbf{A} / c$ is the two-dimensional canonical momentum with vector potential A, $c$ is the speed of light, and $\Delta$ is the strain energy. We employ the Landau gauge and express the vector potential as $\mathbf{A}=(0, B x, 0)$. The Landau level energies then are given as ${ }^{18}$

$$
\begin{aligned}
E_{0}^{\tau_{z}} & =\tau_{z} \Delta \quad \text { for } n=0, \\
E_{n, \lambda}^{\tau_{z}} & =\lambda \sqrt{2 n \hbar^{2} \omega^{2}}+\tau_{z} \Delta \text { for } n \neq 0,
\end{aligned}
$$

with $\lambda= \pm 1$ for the electron and hole bands. The upper $\left(\tau_{z}=\right.$ $+1)$ and lower $\left(\tau_{z}=-1\right)$ surfaces are related by inversion symmetry. Moreover, $\omega=v \sqrt{e B / \hbar}$ is the cyclotron frequency of the Dirac fermions and $n$ is an integer. For zero strain energy, the energy spectrum is similar to that of the ideal Dirac fermions in graphene due to the strong spin-orbit coupling in 
Eq. (1), which distinguishes TIs from a conventional twodimensional electron gas. The energy eigenvalue is $\propto \sqrt{n B}$, which also has been found for the Dirac fermions in graphene. The corresponding eigenfunctions for the upper surface are

$$
\begin{aligned}
& \Psi_{n,+1}^{+1}(r)=\frac{e^{i k_{y} y}}{\sqrt{L_{y}}}\left(\begin{array}{c}
\cos \frac{\theta}{2} \varphi_{|n|-1}\left[\frac{\left(x+x_{0}\right)}{l}\right] \\
-\sin \frac{\theta}{2} \varphi_{|n|}\left[\frac{\left(x+x_{0}\right)}{l}\right]
\end{array}\right), \\
& \Psi_{n,-1}^{+1}(r)=\frac{e^{i k_{y} y}}{\sqrt{L_{y}}}\left(\begin{array}{c}
\sin \frac{\theta}{2} \varphi_{|n|-1}\left[\frac{\left(x+x_{0}\right)}{l}\right] \\
\cos \frac{\theta}{2} \varphi_{|n|}\left[\frac{\left(x+x_{0}\right)}{l}\right]
\end{array}\right)
\end{aligned}
$$

where $L_{y}$ is the dimension of the TI in $y$ direction, $l=\sqrt{\hbar / e B}$, and $n$ is the $n$th Landau level. In addition, $\varphi_{n}\left[\frac{\left(x+x_{0}\right)}{l}\right]=$ $\left(\pi 2^{n} n ! l\right)^{-1 / 2} H_{n}\left(\frac{x+x_{0}}{l}\right) \exp \left[-\frac{1}{2}\left(\frac{x+x_{0}}{l}\right)^{2}\right]$ are the harmonic oscillator eigenstates, $H_{n}(x)$ are the Hermite polynomials, and $\theta=\tan ^{-1}\left(\frac{\sqrt{n} \hbar \omega}{\Delta}\right)$. The eigenstates of the lower surface can be written analogously.

In the presence of a magnetic field there are two contributions to the magnetoconductivity: the longitudinal contribution and the Hall contribution. The former is the localized state contribution which carries the effects of Shubnikov-de Haas $(\mathrm{SdH})$ oscillations. The latter is the nondiagonal contribution. In order to calculate the electrical conductivity in the presence of substrate induced strain and a perpendicular magnetic field, we will follow the formulation of Refs. 20 and 21, which is derived from the general Liouville equation. ${ }^{22,23}$

To obtain the collisional contribution to the conductivity, we assume that the electrons are elastically scattered by randomly distributed charged impurities. It has been shown in Refs. 22 and 23 that charged impurities play a key role in the transport properties of a TI near the nonideal Dirac point. This type of scattering is dominant at low temperature. If there is no spin degeneracy, the collisional conductivity is given by

$$
\begin{aligned}
\sigma_{x x}= & \frac{e^{2}}{L_{x} L_{y} k_{B} T} \sum_{\xi, \xi^{\prime}} f\left(E_{\xi}\right)\left[1-f\left(E_{\xi^{\prime}}\right)\right] W_{\xi \xi^{\prime}}\left(E_{\xi}, E_{\xi^{\prime}}\right) \\
& \times\left(x_{\xi}-x_{\xi^{\prime}}\right)^{2},
\end{aligned}
$$

where $f\left(E_{\xi}\right)=\left[\exp \left(\frac{E_{\xi}-E_{F}}{k_{B} T}\right)+1\right]^{-1}$ is the Fermi Dirac distribution function, with $f\left(E_{\xi}\right)=f\left(E_{\xi^{\prime}}\right)$ for elastic scattering, $k_{B}$ is the Boltzmann constant, and $E_{F}$ is the chemical potential. $W_{\xi \xi^{\prime}}\left(E_{\xi}, E_{\xi^{\prime}}\right)$ is the transmission rate between the one-electron states $|\xi\rangle$ and $\left|\xi^{\prime}\right\rangle$, and $e$ is the charge of the electron. Conduction occurs by transitions through spatially separated states from $x_{\xi}$ to $x_{\xi^{\prime}}$, where $x_{\xi}=\langle\xi|x| \xi\rangle$.

Collisional conductivity arises as a result of migration of the cyclotron orbit due to scattering by charged impurities. The scattering rate is derived analogously to Ref. 24 as

$$
\begin{aligned}
\sigma_{x x}= & \frac{e^{2}}{h} \frac{2 N}{l^{2} \hbar \omega k_{B} T} \frac{e^{4}}{4 \pi^{2} \epsilon_{0}^{2} \epsilon_{r}^{2} k_{0}^{2}} \sum_{n, \tau_{z}}\left[(2 n+1) \cos ^{4} \frac{\theta}{2}\right. \\
& \left.+(2 n-1) \sin ^{4} \frac{\theta}{2}\right] f\left(E_{n, \lambda}^{\tau_{z}}\right)\left[1-f\left(E_{n, \lambda}^{\tau_{z}}\right)\right],
\end{aligned}
$$

where $k_{0}$ is the screening wave vector and $f\left(E_{n, \lambda}^{\tau_{z}}\right)=$ $\left[\exp \left(\frac{\lambda \sqrt{2 n \hbar^{2} \omega^{2}}+\tau_{z} \Delta-E_{F}}{k_{B} T}\right)+1\right]^{-1}$. Therefore, we have

$$
\sigma_{x x} \propto \sum_{n, \tau_{z}} \frac{1}{k_{B} T} f\left(E_{n, \lambda}^{\tau_{z}}\right)\left[1-f\left(E_{n, \lambda}^{\tau_{z}}\right)\right] .
$$

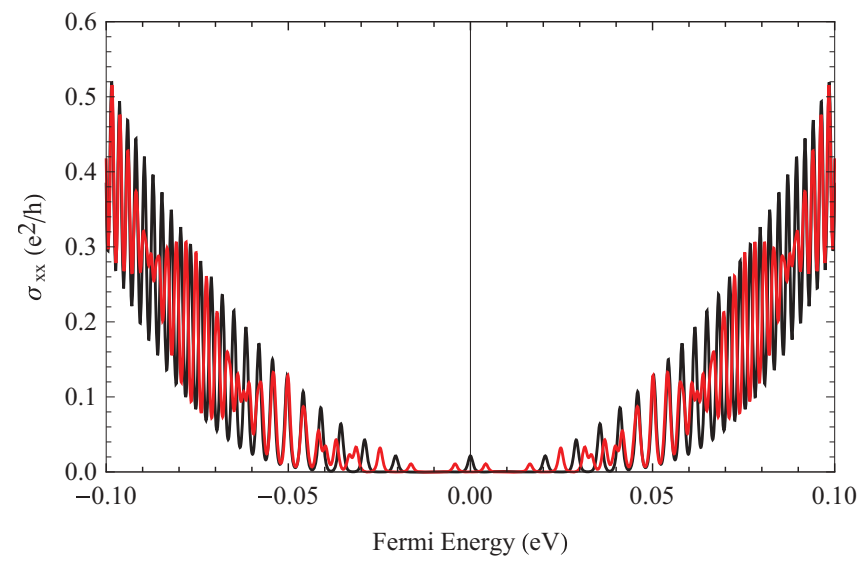

FIG. 1. (Color online) Collisional conductivity as a function of Fermi energy for zero (black lines) and finite (red lines) strain energy. The velocity is taken to be $4 \times 10^{5} \mathrm{~m} / \mathrm{s}, B=2 \mathrm{~T}, T=5 \mathrm{~K}$, and $\Delta=4.2 \mathrm{meV}$.

The origin of the $\mathrm{SdH}$ oscillations in the conductivity is the distribution function entering Eq. (6). When the strain energy is zero we have a symmetric electron-hole spectrum of the magnetoconductivity with a single peak at the charge neutrality point (CNP). This situation is the same as for the Dirac fermions in graphene and different from a conventional two-dimensional electron gas. A closer analytical examination of Eq. (6) shows that the oscillatory part of the conductivity follows the $\mathrm{SdH}$ frequency. There is a $\pi$-Berry phase difference to the two-dimensional electron gas and no difference to the ideal Dirac fermions in graphene. ${ }^{20,21}$ The collisional conductivity is shown graphically in Fig. 1 as a function of the Fermi energy for fixed values of the magnetic field and temperature. The parameters used in our figures are: ${ }^{17-19}$ $N=1 \times 10^{15} \mathrm{~m}^{-2}, \mu_{B}=5.788 \times 10^{-5} \mathrm{eV} / \mathrm{T}, n_{e}=4 \times 10^{15}$ $\mathrm{m}^{2}, k_{0}=10^{-7} \mathrm{~m}^{-1}, v=4 \times 10^{5} \mathrm{~m} / \mathrm{s}, \Delta=4.2 \mathrm{meV}$, and $\epsilon_{r}=4$.

Figure 1 reflects the periodicity of the $\mathrm{SdH}$ oscillations for zero (black lines) and finite (red lines) strain energy. We find a single peak around the CNP for zero strain energy, which confirms a half electron and half hole behavior and is consistent with magnetic response and transport results for graphene. ${ }^{20,21}$ When the strain energy is nonzero, a gap opens at the CNP and a well resolved beating pattern of $\mathrm{SdH}$ oscillations appears away from the CNP. The amplitude of the beating can be increased by increasing the strain energy by means of the substrate. We note that strain alone cannot open a gap at the Dirac point, since it is a time-reversal invariant perturbation. The effects of strain in this work are possible due to the presence of the magnetic field, that breaks time reversal by itself. Furthermore, in the limit of low temperature or high magnetic field, Eq. (6) yields for the collisional conductivity at the CNP the temperature dependence

$$
\sigma_{x x} \propto \frac{1}{k_{B} T} \exp \left(-\frac{2 \Delta}{k_{B} T}\right),
$$

which represents an activated behavior. The width of the gap increases with the strain energy according to Eq. (7), and the collisional conductivity exhibits a gap around the CNP due to 
the splitting of the zeroth Landau level for finite strain. The transition from a maximum to a minimum in the conductivity corresponds to the change form zero to finite strain energy.

The Hall conductivity $\sigma_{x y}$ is obtained from the nondiagonal elements of the conductivity tensor as $\mathrm{s}^{20-23}$

$$
\begin{aligned}
\sigma_{x y}= & \frac{i \hbar e^{2}}{L_{x} L_{y}} \sum_{\xi \neq \xi^{\prime}} f\left(E_{\xi}\right)\left[1-f\left(E_{\xi^{\prime}}\right)\right]\left\langle\xi\left|v_{x}\right| \xi^{\prime}\right\rangle\left\langle\xi^{\prime}\left|v_{y}\right| \xi\right\rangle \\
& \times \frac{1-\exp \left(\frac{E_{\xi}-E_{\xi^{\prime}}}{k_{B} T}\right)}{\left(E_{\xi}-E_{\xi^{\prime}}\right)^{2}} .
\end{aligned}
$$

Following Ref. 24 this simplifies to

$$
\begin{aligned}
\sigma_{x y}= & \frac{e^{2}}{2 \pi \hbar} \sum_{n, \tau_{z}} \sin ^{2} \theta\left(n+\frac{1}{2}\right)\left[f\left(E_{n,+1}^{\tau_{z}}\right)-f\left(E_{n+1,+1}^{\tau_{z}}\right)\right. \\
& \left.+f\left(E_{n,-1}^{\tau_{z}}\right)-f\left(E_{n+1,-1}^{\tau_{z}}\right)\right] .
\end{aligned}
$$

There are two major differences between graphene and TIs: First, we have included strain effects in the presence of a magnetic field, which polarize all Landau levels. Second, the spin and valley degeneracy factor 4 is missing in the prefactor for TIs. This shows that surface states of TIs have a strong spin texture, which is distinct from the ideal Dirac fermions in graphene, and has half the value of the quantum Hall conductivity. Our results show a twofold degeneracy (fourfold for the Dirac fermions in graphene), which confirms the Dirac cone structure of two-dimensional TI surface states.

In the limit of zero temperature and strain energy, Eq. (9) can be further simplified to

$$
\sigma_{x y}=\frac{2 e^{2}}{h}\left(n+\frac{1}{2}\right) .
$$

This result resembles the integer quantum Hall effect in graphene, ${ }^{20,21}$ where the factor 2 is due to surface degeneracy. According to Eq. (10), plateaus appear at $\pm 1, \pm 3, \pm 5, \ldots$, which is exactly what was found in $\mathrm{HgTe}$ transport experiments. ${ }^{17,18}$

In order to compare our theoretical findings with these experiments, the collisional and Hall resistivities are shown in Fig. 2 as a function of the magnetic field for a fixed value of the temperature $(1 \mathrm{~K})$ and a finite strain energy $(4.2 \mathrm{meV})$. The collisional resistivity can be calculated by the relation $\rho_{x x}=\left(B^{2} / e^{2} n_{e}^{2}\right) \sigma_{x x}$ (Refs. 22 and 23), where $n_{e}$ is the electron concentration. We observe extra plateaus in the Hall resistivity due to the substrate induced strain energy. We find that steps between plateaus coincide with sharp peaks of the collisional resistivity. In the limit of high magnetic field, we find a significant splitting of the Hall plateaus and the corresponding peaks in the collisional resistivity. Whereas in the regime of low magnetic field, we observe a quenching of the $\mathrm{SdH}$ oscillations due to the strain energy. Basically, this quenching is a beating pattern of $\mathrm{SdH}$ oscillations. The strain breaks the inversion symmetry, and the surface Dirac fermions of the TI acquire a mass due to the strain energy. These results are in good agreement with the recent experimental observation of strain effects. ${ }^{17,18}$ To clarify the beating patterns in Figs. 1 and 2, we turn to the low magnetic field regime.

We show the collisional resistivity as a function of magnetic field for finite temperature $(1 \mathrm{~K})$ and strain energy $(4.2 \mathrm{meV})$ in

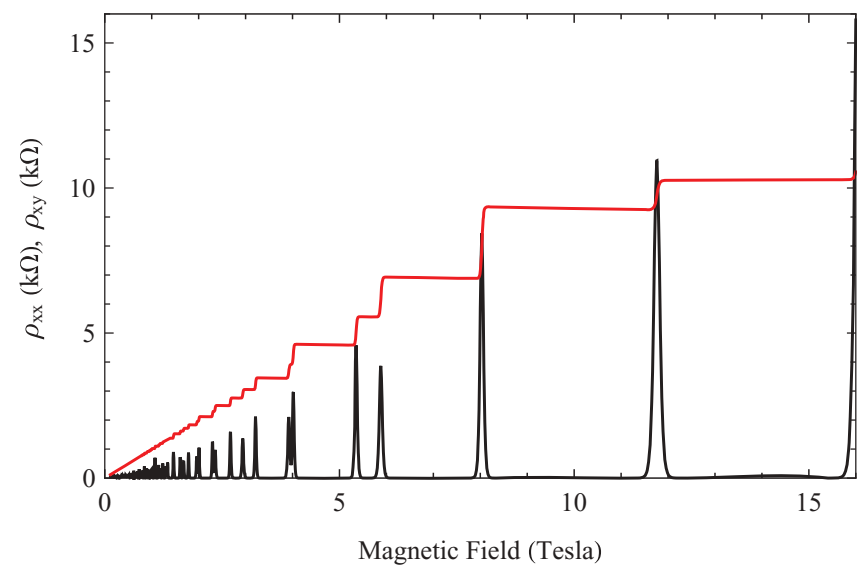

FIG. 2. (Color online) Collisional (black lines) and Hall (red lines) resistivities as a function of magnetic field for a fixed value of the strain energy. The velocity is taken to be $4 \times 10^{5} \mathrm{~m} / \mathrm{s}$, the Fermi energy to be $54 \mathrm{meV}, T=1 \mathrm{~K}$, and $\Delta=4.2 \mathrm{meV}$.

Fig. 3. The beating appears when the subband broadening is of the order of the Landau level separation. In the high magnetic field regime, the strain effects weaken, and the beating pattern is replaced by a splitting of the magnetoconductivity peaks, which persist due to the finite strain energy. To understand the observed beating pattern, we study the phenomenon analytically. In the limit of zero temperature, the collisional conductivity given in Eq. (6) can be written as a $\delta$ function:

$$
\sigma_{x x} \propto \frac{1}{2 \pi l^{2}} \sum_{n, \tau_{z}, \lambda} \delta\left(E_{F}-E_{n, \lambda}^{\tau_{z}}\right) .
$$

Assuming a Gaussian broadening of the Landau levels, this can be expressed as

$$
\sigma_{x x} \propto \frac{1}{2 \pi l^{2}} \sum_{n, \tau_{z}, \lambda} \frac{1}{\Gamma \sqrt{2 \pi}} \exp \left[-\frac{\left(E_{F}-E_{n, \lambda}^{\tau_{2}}\right)^{2}}{2 \Gamma^{2}}\right],
$$

where $\Gamma$ is the width of the Gaussian broadening. Following Refs. 20, 21 and 25, the Poissonian summation formula allows

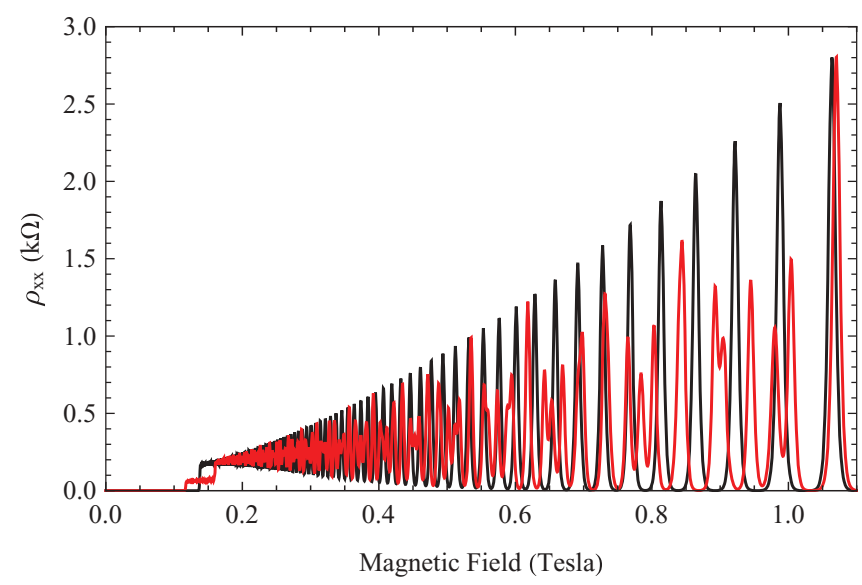

FIG. 3. (Color online) Collisional resistivity as a function of magnetic field, using the same parameters as in Fig. 2. 
us to write the oscillatory part of the conductivity as

$$
\sigma_{x x} \propto \cos \left[\frac{2 \pi E_{F}}{\hbar^{2} \omega^{2}}\left(E_{F} \pm 2 \Delta\right)\right] .
$$

This equation gives the frequency of the $\mathrm{SdH}$ oscillations and the positions of the minima and maxima of the beating pattern. The existence of a beating pattern in a low magnetic field and its disappearance from a higher field is related to the fact that the difference between the two frequencies depends on the strain energy. The nodes occur for $\cos \left[\frac{2 \pi E_{F}}{\hbar^{2} \omega^{2}}\left(E_{F}+2 \Delta\right)\right]+$ $\cos \left[\frac{2 \pi E_{F}}{\hbar^{2} \omega^{2}}\left(E_{F}-2 \Delta\right)\right]=0$. Because this sum is proportional to $\cos \left(\frac{4 \pi E_{F} \Delta}{\hbar^{2} \omega^{2}}\right)$, nodes in the beating pattern occur for $\frac{4 E_{F} \Delta}{\hbar^{2} \omega^{2}}=$ $\pm 0.5, \pm 1.5$, etc.

The spin splitting can be expanded in terms of the magnetic field. It is clear that at a low magnetic field we obtain a constant and there is no beating. For increasing $B, \frac{4 E_{F} \Delta}{\hbar^{2} \omega^{2}}$ decreases so that the collisional conductivity oscillates, exhibiting the beating and splitting of the $\mathrm{SdH}$ peaks at low and high magnetic fields, respectively. Accordingly, we can conclude that the strain is responsible for the formation of the beating pattern and splitting of the $\mathrm{SdH}$ peaks in transport measurements. ${ }^{17,18}$ The former appears in the low and the latter in the high magnetic field regime, which is consistent with Figs. 1, 2, and 3.

\section{CONCLUSION}

In conclusion, we have investigated the strain effects on the quantum magnetotransport properties for the surface states of TIs at finite temperature and magnetic field. When the subband broadening is much smaller than the Landau level separation, the strain manifests itself as a splitting of the $\mathrm{SdH}$ peaks. For a weak magnetic field, when the level broadening is comparable to the Landau level separation, we observe a beating pattern in the magnetoconductivity as a function of the magnetic field. Furthermore, the nonideal Dirac-like spectrum of the HgTe TI surface states exhibits an integer quantum Hall effect with and without a plateau at the CNP for finite and zero strain energy, respectively. A minimum in the longitudinal conductivity leads to a plateau in the Hall conductivity together with a thermally activated behavior. These results are in excellent agreement with recent experiments on the quantum magnetotransport of the surface states of HgTe. *udo.schwingenschlog1@kaust.edu.sa

${ }^{1}$ C. L. Kane and E. J. Mele, Phys. Rev. Lett. 95, 226801 (2005).

${ }^{2}$ B. A. Bernevig, T. L. Hughes, and S. C. Zhang, Science 314, 1757 (2006).

${ }^{3}$ M. König, S. Wiedmann, C. Brüne, A. Roth, H. Buhmann, L. Molenkamp, X.-L. Qi, and S.-C. Zhang, Science 318, 766 (2007).

${ }^{4}$ L. Fu and C. L. Kane, Phys. Rev. B 76, 045302 (2007).

${ }^{5}$ L. Fu, C. L. Kane, and E. J. Mele, Phys. Rev. Lett. 98, 106803 (2007).

${ }^{6}$ J. E. Moore and L. Balents, Phys. Rev. B 75, 121306 (2007).

${ }^{7}$ D. Hsieh, D. Qian, L. Wray, Y. Xia, Y. S. Hor, R. J. Cava, and M. Z. Hasan, Nature (London) 452, 970 (2008).

${ }^{8}$ H. Zhang, C.-X. Liu, X.-L. Qi, X. Dai, Z. Fang, and S.-C. Zhang, Nat. Phys. 5, 438 (2009).

${ }^{9}$ Y. Xia, D. Qian, D. Hsieh, L. Wray, A. Pal, H. Lin, A. Bansil, D. Grauer, Y. Hor, R. Cava, and M. Z. Hasan, Nat. Phys. 5, 398 (2009).

${ }^{10}$ Y. L. Chen, J. G. Analytis, J. H. Chu, Z. K. Liu, S. K. Mo, X. L. Qi, H. J. Zhang, D. H. Lu, X. Dai, Z. Fang, S. C. Zhang, I. R. Fisher, Z. Hussain, and Z. X. Shen, Science 325, 178 (2009).

${ }^{11}$ M. Z. Hasan and C. L. Kane, Rev. Mod. Phys. 82, 3045 (2010).

${ }^{12}$ J. E. Moore, Nature (London) 464, 194 (2010).
${ }^{13}$ A. Roth, C. Brüne, H. Buhmann, L. W. Molenkamp, J. Maciejko, X.-L. Qi, and S.-C. Zhang, Science 325, 294 (2009).

${ }^{14}$ Y. C. Chang, J. N. Schulman, G. Bastard, Y. Guldner, and M. Voos, Phys. Rev. B 31, 2557 (1985).

${ }^{15}$ O. A. Pankratov, Semicond. Sci. Technol. 5, S204 (1990).

${ }^{16}$ X. Dai, T. L. Hughes, X.-L. Qi, Z. Fang, and S.-C. Zhang, Phys. Rev. B 77, 125319 (2008).

${ }^{17}$ B. Büttner, C. X. Liu, G. Tkachov, E. G. Novik, C. Brüne, H. Buhmann, E. M. Hankiewicz, P. Recher, B. Trauzettel, S. C. Zhang, and L. W. Molenkamp, Nat. Phys. 7, 418 (2011).

${ }^{18}$ C. Brüne, C. X. Liu, E. G. Novik, E. M. Hankiewicz, H. Buhmann, Y. L. Chen, X. L. Qi, Z. X. Shen, S. C. Zhang, and L. W. Molenkamp, Phys. Rev. Lett. 106, 126803 (2011).

${ }^{19}$ W. Shan, H. Lu, and S. Shen, New J. Phys. 12, 043048 (2010).

${ }^{20}$ V. P. Gusynin and S. G. Sharapov, Phys. Rev. B 71, 125124 (2005).

${ }^{21}$ V. P. Gusynin and S. G. Sharapov, Phys. Rev. Lett. 95, 146801 (2005).

${ }^{22}$ M. Charbonneau, K. M. Van Vliet, and P. Vasilopoulos, J. Math. Phys. 23, 318 (1982).

${ }^{23}$ P. Vasilopoulos, Phys. Rev. B 32, 771 (1985).

${ }^{24} \mathrm{M}$. Tahir and U. Schwingenschlögl (unpublished).

${ }^{25}$ Z. Tan, C. Tan, L. Ma, G. T. Liu, L. Lu, and C. L. Yang, Phys. Rev. B 84, 115429 (2011). 